\title{
EVALUATION MODEL OF LIGHTING ENVIRONMENT FOR SUBWAY STATION SPACE BASED ON BACK-PROPAGATION NEURAL NETWORK
}

\author{
Wenhao DUN \\ School of Civil Engineering \& Architecture of Wuhan University of Technology / Hubei \\ Institute of Fine Arts, 430000, Wuhan, Hubei, China; \\ E-mail:26978991@qq.com
}

\begin{abstract}
BP neural network is a new computer technology, which has natural advantages in the study of lighting environment evaluation model of subway station space. With the implementation of energy conservation and emission reduction, how to establish a suitable calculation model for the lighting environment of subway station space is also a hot research field. Firstly, the development trend of subway and the research status at home and abroad is introduced, then the specific application method of BP neural network is expounded, the evaluation criteria and evaluation model of space lighting environment of subway station is established. BP neural network is used to establish the corresponding mathematical model, through the weight calculation, the appropriate evaluation system is established, and finally the model is used to verify it.

Keywords: BP neural network, subway station, lighting environment evaluation, intelligent algorithm

\section{INTRODUCTION}

The development of the subway is a far-reaching theme, Nowadays, the comfort and convenience of travel is emphasized in the whole world, and the interior lighting environment design of metro also plays an important role in the overall comfort design and development of metro [1]. At present, China's metro is mostly imported or jointly
\end{abstract}

developed from other countries in the interior lighting environment design of trains, foreign lighting design is mainly used, and the lighting reference value and evaluation basis conforming to China's national visual environment are lacking [2]. At that same time, the design only bases on meet physical parameters such as illuminance and the like in lighting design, lacks subjective feel evaluation on the visual environment of the high-speed train's passengers. In particular to the visual psychology scale of "visual brightness”, “ visual illuminance”, “ visual concentration point" that sort of things [3]. There is a lack of reasonable consideration on the colour temperature of the light source of subway indoor lighting, only using the evaluation method which accords with the colour temperature feeling of foreign passengers. The problems of high physical il-

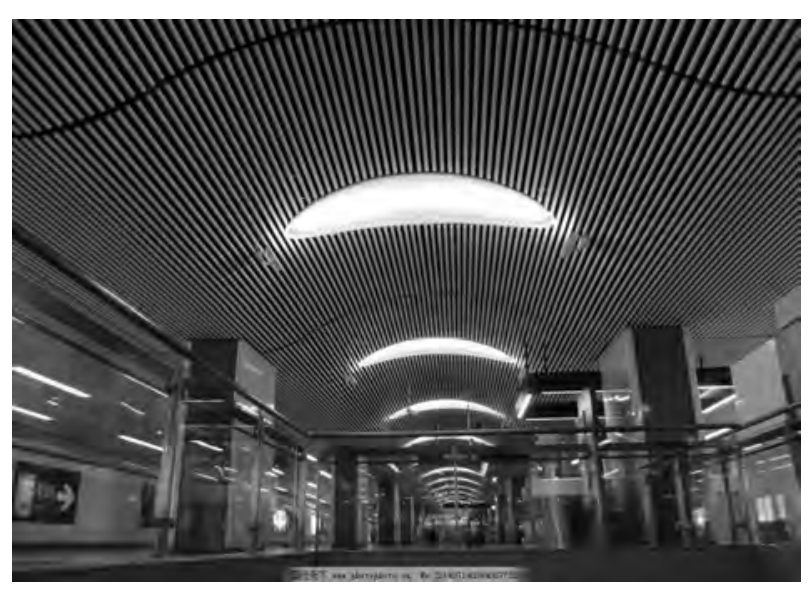

Fig.1. Lighting environment of a subway station 
lumination and poor actual illumination effect exist in the structural design, installation mode and spatial scale of the lamp, in particular, the problem of the space installation scale of the indirect lighting device leads to the problems of large brightness contrast of the light source, uncomfortable glare and so on [4]. Therefore, the research of this paper has important practical significance to solve the problem of interior lighting environment design of subway in China. At the same time, the research on this aspect can provide a reference for designers, which has a strong practical significance [5].

\section{START OF THE ART}

BP neural network is an artificial intelligence algorithm, at the beginning of the $20^{\text {th }}$ century, people studied the layout and structure of the animal brain, found that the brain is made up of a large number of neurons connected to each other. Complex non-linear information can be processed in parallel in such systems, known as neural networks [6]. Through the simulation and reference of animal neural network, scientists put forward the concept of artificial neural network [7]. The essence of artificial neural network is an algorithm model, like animal brain, artificial neural network is composed of a large number of artificial neurons [8]. The structure and function of these artificial neurons are very simple, but they can be connected to each other and form a network to achieve very powerful functions [9]. Artificial neural network can learn independently, self - perfect, and process complex nonlinear information in parallel. It has a very broad application prospect in solving complex problems such as thinking, reasoning and consciousness [10]. Therefore, it is of great significance to use BP neural network algorithm to study the evaluation model of lighting environment in subway station space.

\section{METHODOLOGY}

\subsection{Evaluation Criteria for Spatial Lighting Environment of Subway Stations}

The evaluation of space lighting environment of subway station under fluorescent lighting is mainly based on the function of lighting. The main purpose of the lighting is to meet the physical characteristics of the light environment required by space users to accurately identify the surrounding envi- ronment and the route to the subway station space. And LED has the characteristics of high light efficiency, long life, adjustable light, which is applied to the lighting environment created by subway station space lighting. Besides meeting the functional requirements, its energy-saving characteristics are more prominent. Therefore, in order to study the evaluation system suitable for subway station space lighting environment, it is necessary to combine the characteristics of lighting function and energy saving to conduct in-depth study, the theoretical model of spatial lighting environment evaluation system for metro station is constructed. The weight of each index in the model is solved by particle swarm optimization, and the advantages and disadvantages of lighting environment under two kinds of light sources are obtained from the model and field investigation. A subway station lighting environment is shown in Fig. 1.

\subsection{Subway Station Spatial Lighting Environment Evaluation Model}

The evaluation index of space lighting environment of subway station mainly includes two kinds of indexes: functional index and energy-saving index, among which the functional index is divided into two categories: light environment and space environment. The light environment factors include illuminance level, illuminance uniformity, luminance distribution, glare index, colour temperature, colour rendering index, illumination induction, recognition, etc. Lighting energy saving includes lighting power density (LPD) value, energy saving lamps and lanterns, new energy utilization. The evaluation index system for evaluating the spatial lighting environment of the subway station is obtained after three rounds of expert consultation by using the Delphi method, in which a total of 16 indicators are included: illuminance level, illuminance uniformity, luminance level, luminance distribution, glare, colour temperature, colour rendering, illumination induction, recognition, stereoscopic impression, environmental coordination, illumination artistry, control strategy, control mode, LPD, energy-saving lamps and lanterns, The analytic hierarchy process is used to decompose a complex decision-making problem into several interrelated levels. The hierarchical structure diagram of spatial lighting environment evaluation of metro station is shown in Fig. 2. 


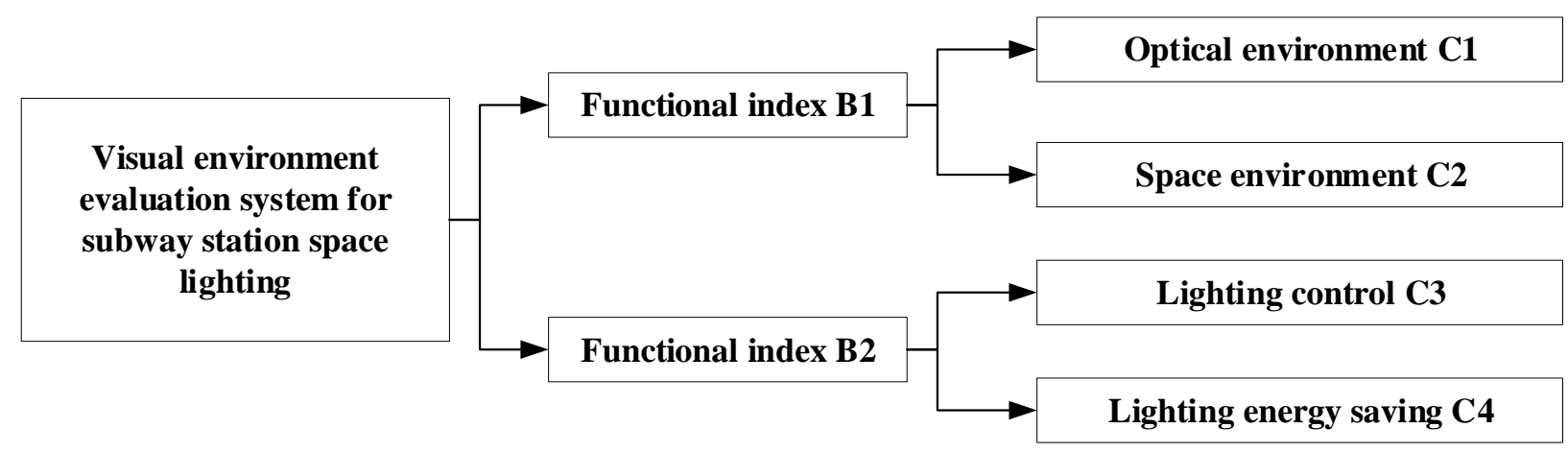

Fig.2. Hierarchical structure of subway station space visual environmental assessment

\subsection{BP Neural Network Model}

The interconnections between artificial neurons form is a directed graph, and the structural diagram of artificial neurons is shown in Fig. 3.

The corresponding real number for each connection is referred to as the connection weight; the set of weights may be considered as longterm memory. There are two main types of connection weights, excitation and suppression, positive connection weights for excitation and negative connection weights for suppression. The connection of weights is the feature description of artificial neural network. At time $t$, the corresponding value of the artificial neuron is called the state of the artificial neuron, also called the excitation value of the artificial neuron, if a certain artificial neuron is represented by $u_{j}$, then its state is represented by $x$, and $X(t)$ represents a set of neuronal states of a certain amount. State set can be expressed by continuous function or discrete function. It can be bounded function or unbounded function. This set may have solution in some real field or finite solution. In practical applications, generally take two values, with 0 and 1 , or -1 and 1 to represent two states, there are some cases need to take the value in the continuous real number. Information is

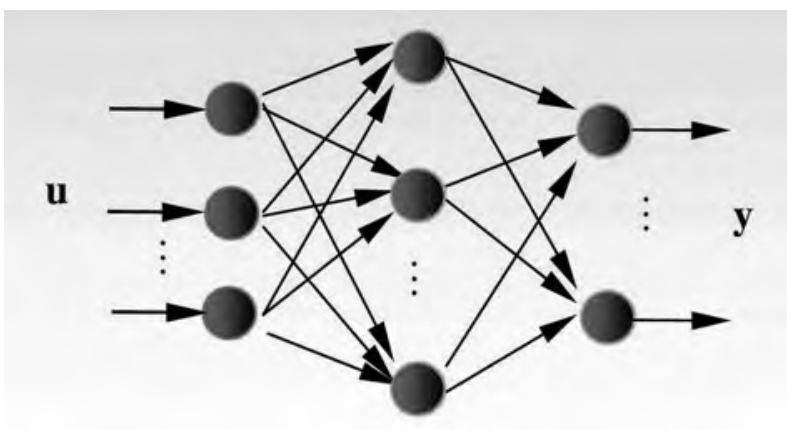

Fig.3. BP neuron structure diagram passed through each neuron to obtain an output signal, which is passed through the connection weights to the other connected neurons. The output signal value depends on the state of the neuron. This interaction is a function of the output transformation from function $f_{j}$ to the neuron $u_{j}$.

The calculation formula of nerve source is presented in equation 1.

$$
Z_{j}(t)=f_{j}\left(x_{j}(t)\right)
$$

where $Z_{j}(t)$ represents the output signal of the neuron $u_{j}$, and $f$ represents a component function.

By grouping artificial neurons in this layer, and taking each neuron group as a whole, the batch control of artificial neurons is realized, and the network operation speed is accelerated. For example, the use of lateral inhibition mechanism to make some artificial neurons in a state of no output, and can have the largest output in the layer of artificial neurons selected.

An interconnected network can connect any two artificial neurons. Belonging to this kind of network is Hopfield network and Boltzmann machine. In networks without feedback, the signal processing function stops as the signal passes through the artificial neuron. Starting from the initial state, the signal will change several times, and finally achieve dynamic balance, the structure and characteristics of the network is different, and finally the reached state is not the same.

The reasonable determination of the weight is the premise of correct evaluation. After determining the comprehensive evaluation index of spatial lighting visual environment of subway station, the weight of each index becomes an important part of the evaluation system research, and its accuracy is more related to the correctness and scientific character of the final evaluation results. Based on this, 
Table 1. Classification Scale for Comparison of Evaluation Indicators

\begin{tabular}{|c|l|c|l|}
\hline Scale value & \multicolumn{1}{|c|}{ Relative comparison } & Scale value & \multicolumn{1}{c|}{ Relative comparison } \\
\hline 1 & A is as important as B & 1 & A is as important as B \\
\hline 3 & A is more important than B tip & $1 / 3$ & A is a little less important than B \\
\hline 5 & A is more important than B & $1 / 5$ & A is not B important \\
\hline 7 & A is much more important than B & $1 / 7$ & A is much less important than B \\
\hline 9 & A is more important than B & $1 / 9$ & A is far from B \\
\hline
\end{tabular}

we intend to use analytic hierarchy process to build hierarchical structure, establish the initial judgment matrix, use particle swarm optimization to solve the index weight, establish the urban subway station spatial lighting environment evaluation system model. The calculation flow is shown in Fig. 4.

Analytic hierarchy process is an effective method to determine the weight vector, when calculating weights by analytic hierarchy process. The function $f(x, y)$ represents the importance scale for the ratio of $x$ to $y$ in general. If $f(x, y)>1, x$ is more important than $y$, otherwise $\mathrm{y}$ is more important than $x$. Table 1 shows the scale values of grade division for comparison of evaluation indicators.

In the current application, the judgment matrix given by the scale of 1-9 is difficult to achieve consistency, so that the judgment matrix is inconsistent with the actual judgment thinking, the calculation distortion of the relative weight is caused. This is also the current multi-decision-making problems need to be solved and challenges. Through the study of the initial judgment matrix, in order to check the consistency of the initial judgment matrix, get a reasonable weight vector, we need to build a proper consistency correction model, and use intelligent algorithm to optimize the solution, quickly get the judgment matrix and the corresponding weight vector can pass the consistency check. The obtained judgment matrix is fed back to the experts to evaluate whether the modified matrix is within an acceptable range in practice and whether a group of weight vectors obtained by solving the nonlinear model can be used as the weight of each evaluation index required to solve the practical problem. If the expert opinion is positive, the modified judgment matrix is acceptable; On the other hand, the parameters need to be reset and solved, again to verify, until recognized by the experts.

The calculation formula of the objective function is shown in equation 2 .

$$
\min Y=\sum_{i=1}^{n} \sum_{j=1}^{n}\left[\lambda_{1}\left(x_{i j}-a_{i j}\right)^{2}+\lambda_{2}\left(x_{i j}-\omega\right)^{2}\right],
$$

where $\lambda$ is the weight value, $a$ is the weight matrix and $y$ is the target function value. The smaller $y$ in the objective function is, the better it is. It shows that the adjusted amplitude is relatively small and the modified matrix quality is higher when the consistency condition is satisfied. Wherein $\lambda_{1}$ and $\lambda_{2}$ are weight factors, and the assignment is mainly determined according to the specific requirements in practice, on the one hand, the compliance degree of the expert judgment matrix, on the other hand,

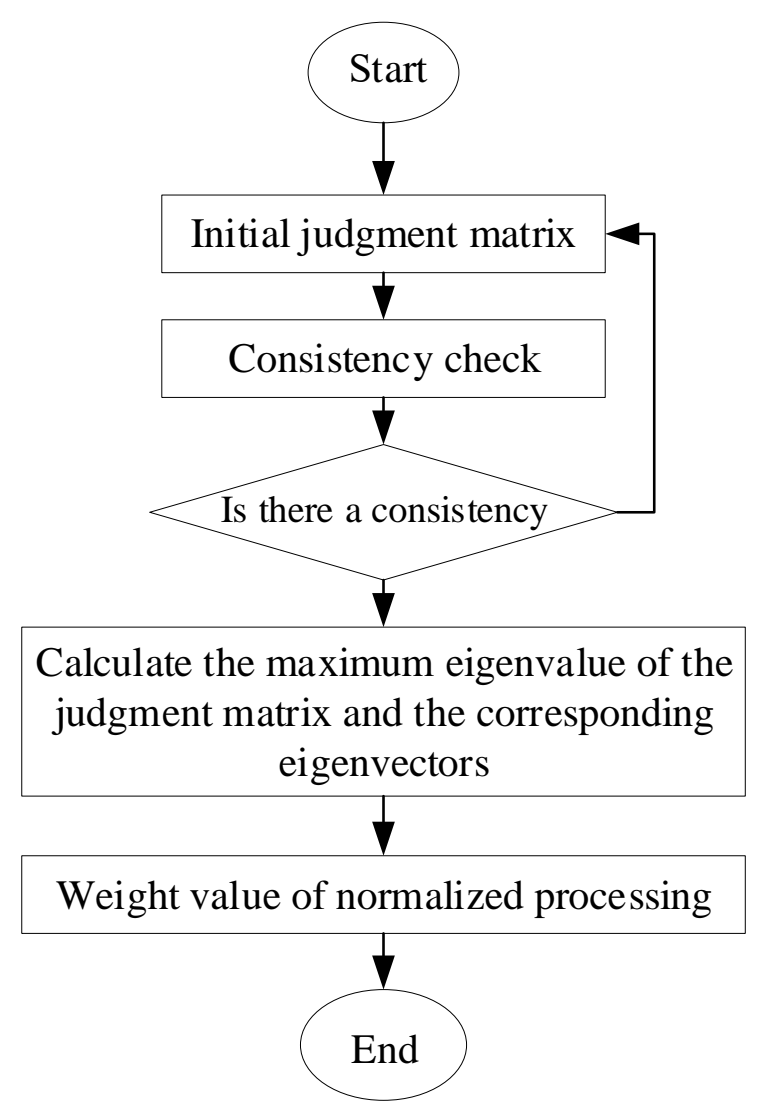

Fig.4. Index weight calculation process 
Table 2. Visual Environmental Assessment System of Subway Station Space

\begin{tabular}{|c|c|c|c|}
\hline Standard layer B & Index layer $\mathbf{C}$ & Evaluation index D & Index weight \\
\hline \multirow{12}{*}{ Functional index B1 } & \multirow{9}{*}{ Optical environment $\mathrm{Cl}$} & Illuminance level D1 & 0.0901 \\
\hline & & Illumination uniformity D2 & 0.0729 \\
\hline & & Luminance level D3 & 0.1015 \\
\hline & & Luminance distribution D4 & 0.1184 \\
\hline & & Glare D5 & 0.0620 \\
\hline & & Colour temperature D6 & 0.0881 \\
\hline & & Chromaticity D7 & 0.0219 \\
\hline & & This visual induction D8 & 0.0743 \\
\hline & & Identifiability D9 & 0.1024 \\
\hline & \multirow{3}{*}{ Space environment C2 } & Stereoscopic D10 & 0.0199 \\
\hline & & Environmental coordination D11 & 0.0139 \\
\hline & & Photoperiod D12 & 0.0124 \\
\hline \multirow{4}{*}{ Energy saving index B2 } & \multirow{2}{*}{ Lighting control C3 } & Screwing strategy D13 & 0.0454 \\
\hline & & Screwing method D14 & 0.0503 \\
\hline & \multirow{2}{*}{ Lighting energy saving C4 } & Lighting power density D15 & 0.0755 \\
\hline & & Energy saving luminaire D16 & 0.0511 \\
\hline
\end{tabular}

the requirement degree of meeting the consistency index. The main goal of the solution of the model is to meet the consistency index, so the degree of meeting the consistency index requirements is higher than the degree of following the expert judgment matrix. In the process of original expert judgment matrix correction for each element adjustment of a constraint index, the smaller $\theta$ is the better. $A_{i j}$ is the element of initial judgment matrix, $x_{i j}$ and $w_{i}$ are the elements of judgment matrix with good consistency and corresponding index weights.

After a lot of research, BP neural network algorithm and particle swarm optimization are combined to solve the least squares consistency correction model of analytic hierarchy process, so, as to obtain the judgment matrix with good consistency. Revise the index weight from both subjective and objective aspects to improve the accuracy of the index weight. The basic principle is that there are $\mathrm{M}$ particles in $\mathrm{D}$ - dimensional space, the motion space of particles is the solution space, the objective function value to be optimized is the fitness of particles, the position vector of particles represents the variables in the solution space of optimi- zation problems, and the motion process of particles is the search process of solutions.

The formula for calculating the particle position is shown in equation 3 :

$$
x_{i}=\left(x_{i 1}, x_{i 2}, \ldots, x_{i D}\right) .
$$

The calculation formula of particle velocity is shown in equation 4.

$$
v_{i}=\left(v_{i 1}, v_{i 2}, \ldots, v_{i D}\right)
$$

The formula for calculating the optimal position is shown in equation 5 .

$$
G^{0}=\min \left\{f\left(P_{1}^{0}\right), \ldots, f\left(P_{i}^{0}\right), \ldots, f\left(P_{M}^{0}\right)\right\},
$$

where $G$ is the optimal position of matrix.

\subsection{The Construction of Subway Station Space Lighting Environment Evaluation System}

The judgment matrix and the sorting weight vector obtained by the particle swarm optimiza- 


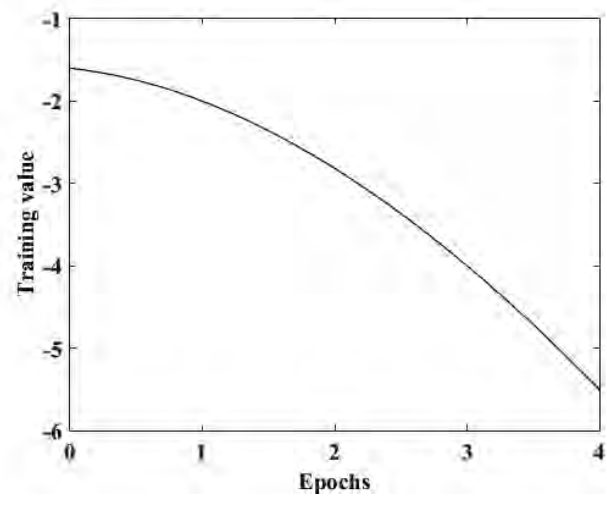

Fig.5. First class parameter training diagram

tion algorithm are fed back to the experts of the initial judgment matrix, and the experts believe it is acceptable. Therefore, the modified judgment matrix is taken as the final judgment matrix, and the weight vector obtained by solving the model is taken as the weight of each evaluation index, thus the comprehensive evaluation model of lighting environment in subway station space is constructed. As shown in Table 2.

\section{RESULT ANALYSIS AND DISCUSSION}

The model-based inspection and evaluation method is a method based on the design process, mainly through lighting simulation design software. Its advantages are easy operation, fast and reliable acquisition of index data similar to the actual project. Foreign mature professional software: AGI32, DIALux, LightStar, Lummenmicro, Autolux, Inspire, etc. DIAlux is mainly used for inspection, mainly because the software support Chinese, easy to operate, easy to master, and pay attention to the accuracy of the calculation, lighting error is only $3 \%$ $7 \%$. There are many manufacturers supporting optional lighting libraries, which can support the calculation of light environment and three-bit light distribution simulation diagram in different scenes, can output including illuminance, brightness value contour, data analysis report and lamps and lanterns of light distribution, unified glare index, lighting scene power density and other data. The model-based in-

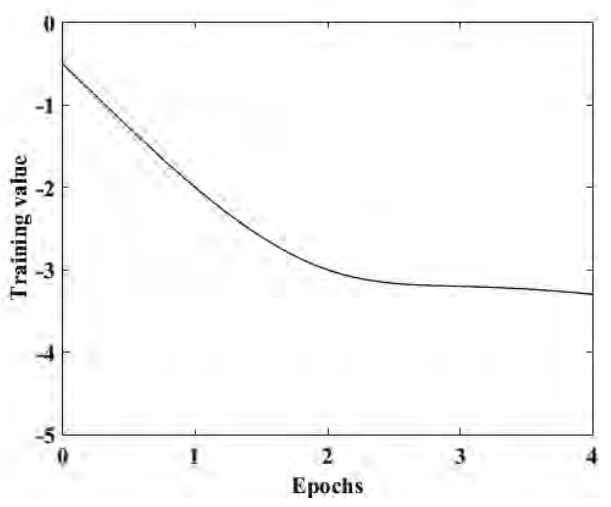

Fig.6. Second class parameter training diagram

spection and evaluation method is mainly realized: detecting the power consumption of large-space lighting scheme and the implement ability of lighting effect, then a metro model of a standard island platform layer based on foundation structure in China is studied.

The learning of sample pattern $P$ by neural network is to minimize $E$. In the secondary test of the index, the evaluation data (experts' evaluation) obtained by the Delphi method is taken as a training set to form a matrix of $P$, The $E$ value is taken as a matrix of $T$ formed by training samples, the number of input layers is $M$ of parameters, the number of output nodes is 1 , and the number of hidden layers is $L=2 m+1$. And set the learning accuracy $e=$ 0.0001 , training times $n=1800$, weight adjustment parameter $\alpha=0.5$, offset adjustment parameter $\beta$ $=0.8$.

The training results of the primary parameters are shown in Fig. 5, and the values of the results are shown in Table 3.

The training results of the secondary parameters are shown in Fig. 6, and the values of the results are shown in Table 4.

The use of BP neural network method is mainly to test the relationship between the relatively complex two to four parameters, Fig. 5 shows that due to the number of first-order parameters input is small, the number of training steps is very small. On that other hand, the high value of the primary parameter e can be used as a reference only in the

Table 3. Level BP Parameters Training Results and Delphi $E$ Value Comparison Table

\begin{tabular}{|c|c|c|c|}
\hline Parameter & Design process & Electricity consumption measurement & Lighting effect \\
\hline The training results & 0.8998 & 0.7385 & 0.7973 \\
\hline Expected output & 0.9000 & 0.7400 & 0.8000 \\
\hline
\end{tabular}


Table 4. Two Parameters of BP Training Results and Delphi $E$ Value Comparison Table

\begin{tabular}{|c|c|c|c|c|c|c|c|c|}
\hline 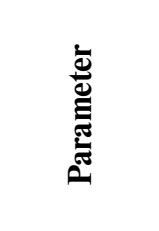 & 童 & 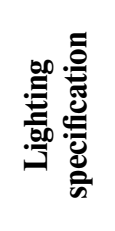 & 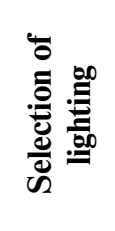 & 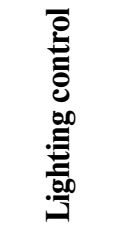 & 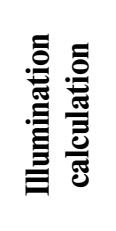 & 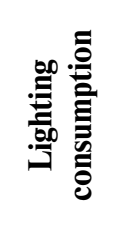 & 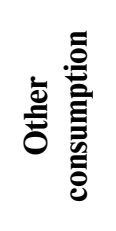 & 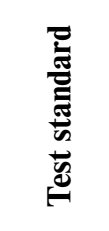 \\
\hline $\begin{array}{l}\text { Training } \\
\text { results }\end{array}$ & 0.3192 & 0.8783 & 0.8795 & 0.8056 & 0.7407 & 0.8466 & 0.5198 & 0.7591 \\
\hline $\begin{array}{l}\text { Expected } \\
\text { output }\end{array}$ & 0.3200 & 0.8800 & 0.8800 & 0.8000 & 0.7400 & 0.8400 & 0.5200 & 0.7600 \\
\hline
\end{tabular}

case where it is determined as an index. The capacity grade of the secondary parameter is more in line with the standard of BP neural network method. By processing a large number of data, the BP training result $E$ value is compared with the expected output E value of Delphi method, that is, the correctness of the previous index screening is verified by using objective data.

The results from the above table show that the $e$ value trained by BP neural network method is basically consistent with the $e$ value calculated by Delphi method. The relative error range of secondary parameters is $0.11 \% \sim 0.78 \%$, and all of them are within the allowable error range. Therefore, it can be comprehensively determined that the previously determined index set is feasible and accurate. The index parameters of secondary confirmation were consistent with those of Delphi method. The number and quality of learning samples affect the learning performance of neural network model to a great extent. The samples selected in our research are slightly less, but the results are still more accurate after calculation. Therefore, if we increase the number of learning samples on this basis, we will achieve more accurate and good results.

\section{CONCLUSION}

With the development of computer technology, it has been widely applied to some problems in real life environment. Therefore, aiming at the problem of comprehensive evaluation of lighting environment in metro station space, this paper uses the theoretical knowledge of light environment, environmental psychology and fuzzy mathematics to construct the theoretical model of comprehensive evaluation of lighting environment in metro station space. The model is applied to the comprehen- sive evaluation of the lighting environment of the subway station space of Shenzhen metro line 2 illuminated by LED light sources and Shenzhen metro line 3 illuminated by fluorescent lamps. According to the questionnaire of each index on the spot and the model, the optimal lighting environment is obtained, the rationality of the selected index in the model and the validity of the index weight are verified by combining the subjective investigation result and the model theoretical data. Thus, the evaluation model has universal applicability, provides model reference for the future evaluation of subway station space lighting environment, and provides theoretical basis for subway station space lighting design. Indoor lighting environment is a multi-factor comprehensive lighting environment, and the field of indoor environment lighting, which is dominated by artistic lighting, needs further study.

\section{REFERENCES}

1. Englund S R, O’brien J J, Clark D B. Evaluation of digital and film hemispherical photography and spherical densiometry for measuring forest light environments. Canadian Journal of Forest Research, 2000. V30, \#12, pp.1999-2005.

2. Poorter L, Arets E J M M. Light environment and tree strategies in a Bolivian tropical moist forest: an evaluation of the light partitioning hypothesis. Plant Ecology, 2003. V166, \#2, pp.295-306.

3. Hernandez Duran T, Ravela N, Sanchez Rivero $\mathrm{S}$, et al. Evaluation of Different Light Conditions in the Working Environment for Handling Photosensitive and Thermolabile Compounds. Journal of AOAC International, 2015. V98, \#6, pp.1491-1495.

4. Mills P R, Tomkins S C, Schlangen L J M. The effect of high correlated colour temperature office lighting 
on employee wellbeing and work performance. Journal of circadian rhythms, 2007. V5, \#1, p.2.

5. Haiying Li, Xian Li, Xinyue Xu, Jun Liu, Bin Ran. Modeling departure time choice of metro passengers with a smart corrected mixed logit model - A case study in Beijing. Transport Policy. 2018, \#69, pp.106-121.

6. Li D H W, Lam J C. Evaluation of lighting performance in office buildings with daylighting controls. Energy and buildings, 2001. V33, \#8, pp.793-803.

7. Iwata T, Tokura M, Shukuya M, et al. A pilot experiment on a method for evaluating acceptability of a daylit luminous environment. Journal of Thermal Biology, 1993. V18, \#5-6, pp.555-559.
8. Xu, X., Xie, L., Li, H., \& Qin, L. Learning the route choice behavior of subway passengers from AFC data. Expert Systems with Applications, 2018. \#95, pp. 324-332.

9. Stansbury J, Mittelsdorf A M. Economic and environmental analysis of retrofitting a large office building with energy-efficient lighting systems. Environmental management, 2001. V27, \#6, pp.909-918.

10. Aghemo C, Fabbri M, Verso V L, et al. Visual perception in aircraft cockpit under real ambient lighting conditions: A procedure for the evaluation of readability and legibility. Perception ECVP abstract, 2009. V38, pp.181-181.

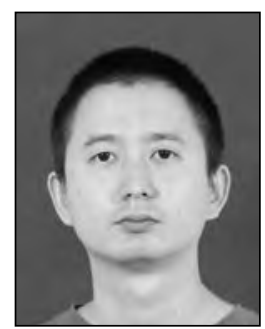

\section{Wenhao DUN,}

on-the-job doctor and lecturer. Graduated from school of civil engineering and architecture, Wuhan university of technology. Work in Wuhan university of technology, research direction is underground engineering lighting design 